\title{
Lo spazio del sacro
}

\author{
di Nicola Righetti ${ }^{1}$
}

\section{Introduzione}

Le ricerche sui luoghi sacri si trovano all'incrocio multidisciplinare tra scienze sociali, geografiche e delle religioni. Sono anche punto di convergenza di due tendenze, teorica l'una, economica l'altra, che aiutano a comprenderne l'importanza. La prima è nota come spatial turn e corrisponde ad una più attenta considerazione del ruolo dello spazio nella vita sociale e individuale. Si è alimentata delle lezioni di Michel Foucault (1986/1967), Henri Lefebvre (1974) e Michel de Certeau (2001/1980), che hanno contribuito a definire un'idea di spazio come territorio socialmente costruito, interpretato, plasmato da interessi, conteso, negoziato (Kilde 2007; Knott 2010). La seconda è rappresentata dal mercato del turismo religioso, che ha nei luoghi sacri il suo principale punto d'attrazione (Timothy, Olsen 2006). Se queste tendenze aiutano a contestualizzare il ruolo dei siti sacri a livello teorico ed economico, due fattori di natura socio-culturale aiutano invece a comprenderne la complessità concettuale. Il primo fattore di complessità è l'estensione del sacro nella sfera extrareligiosa. Le ricerche sugli spazi sacri, infatti, possono riguardare i luoghi delle grandi tradizioni religiose, come città sante, chiese, santuari, ambienti naturali, templi e mete di pellegrinaggio, ma anche monumenti nazionali e altri spazi lontani dall'idea comune di luogo sacro, come residenze e sepolture di idoli della cultura pop e altri luoghi di consumo. Ciò non deve stupire. Il regno del sacro, soprattutto nella società contemporanea, non è rinchiuso tra i confini tracciati dalle istituzioni religiose. Il pluralismo è cresciuto, la società si è secolarizzata e le tradizioni religiose hanno perso autorità sui loro stessi simboli. Gli individui, con la mentalità del consumatore, possono scegliere e assemblare simboli sacri in un bricolage religioso personale (Luckmann 1969/1967; Trombetta 2004), e la spiritualità individuale può andare alla ricerca di luoghi intrisi di valori che rispondono alle proprie esigenze di senso, più che ad una prescrizione dottrinale (Timothy, Olsen 2006: 3ss.). Senz'altro, i siti sacri delle grandi tradizioni religiose sono i più centrali nel panorama globale, ciò nono-

\footnotetext{
${ }^{1}$ Dottore di ricerca in Sociologia e ricerca sociale all’Università di Verona.
} 
stante, i luoghi possono essere sacri perché sacri a Dio, perché sacri alla Nazione, perché sacri all'individuo o a particolari gruppi sociali e culturali. In ogni caso, tutti sono riconosciuti tali perché rinviano ad un immaginario costituito da valori e da ideali che si trovano al cuore di un'identità personale e sociale, perché sono teatro di riti e sono avvolti in narrazioni significative. La seconda condizione di complessità, connessa alla prima, è il pluralismo, sia culturale, sia degli interessi. In virtù della loro importanza economica e, ancor prima, di quella simbolica, i luoghi sacri possono catalizzare contese sulla legittima autorità che ne deve avere il controllo, nonché conflitti relativi alla definizione e ridefinizione dei significati e dei valori che essi rappresentano (Chidester, Linenthal 1995).

Il più o meno ampio focus delle ricerche, così come la maggiore o minore enfasi sulla costruzione sociale dei luoghi e sui conflitti che essi catalizzano, dipendono in parte dalla prospettiva teorica di partenza. Come tutti i fenomeni di una certa complessità, sacro e spazio sacro sono stati variamente interpretati. Nella letteratura si possono identificare due significativi filoni di studio: quello fenomenologico e quello costruzionista. Si tratta di approcci mainstream allo studio del sacro, prima ancora che allo studio dello spazio sacro, e poiché non esiste definizione di spazio sacro che sia indipendente da una definizione di sacro, questa sarà presa preliminarmente in considerazione, per presentare, subito dopo, il concetto generale di spazio sacro e alcune sue caratteristiche alla luce dei due approcci sopraccitati.

\section{Sacro e spazio sacro}

Nella vasta letteratura sul sacro si possono individuare due traiettorie significative: l'una si diparte dalla definizione di sacro di Émile Durkheim (2005/1912), l'altra da quella di Rudolf Otto (2009/1936). Entrambi questi studiosi elaborano le loro teorie in dialogo con l'antropologia dell'epoca (Pignato 2001), ma con intenzioni ed esiti diametralmente opposti. Mentre Durkheim considera il sacro un prodotto della pratica sociale, per Otto è una categoria a priori dello spirito umano, irriducibile e inspiegabile, soltanto descrivibile. Più specificamente, per Durkheim il sacro è un fenomeno di natura emozionale, socialmente prodotto dal rituale collettivo. Per le emozioni estatiche che provoca, il rito collettivo suscita negli aderenti la sensazione di una potenza trascendente che li domina. In realtà, questa sensazione è prodotta dal rituale stesso e dunque dalla collettività. Quest'emozione e il senso di potere che le è connesso, vengono associati ad emblemi materiali e ad idee che ne acquisiscono le caratteristiche e che rappresentano, simbolicamente, l'unità del corpo sociale da cui derivano. Poiché idee, oggetti e luoghi non sono sacri in sé ma per la loro consacrazione sociale, lo sguardo sociologico può spaziare dalle religioni tradizionali al mondo secolare, trovando simboli identitari, valori idealizzati e riti capaci di suscitare effetti analoghi a quelli del rito religioso. La ricerca del sacro nel mondo secolare può approdare, per esempio, allo sport (Prebish 
1984), al consumo (Belk et al. 1989), alla politica (Durkheim 2005/1912: 490s.), sia nella versione totalitaria della "religione politica" (Gentile 2000), che in quella democratica della "religione civile" (Bellah 1967).

La prospettiva del fenomenologo delle religioni Otto è diversa, anche se molti elementi di base sono simili. Egli condivide l'idea che il fattore emotivo del sacro sia prioritario e non ne ignora il significato sociale e morale, ma considera riduttiva ogni sua spiegazione scientifica. Il brivido di sacro timore provocato dalla percezione del mysterium tremendum et fascinans non è provocato da cause esterne, siano esse naturali o sociali, ma da una «predisposizione mentale unica di tal genere, definitivamente difforme da ogni facoltà naturale» (Otto 2009/1936: 30). I fenomeni esterni sono uno stimolo per l'apparizione del fenomeno sacrale, ma non la loro causa. La descrizione delle caratteristiche essenziali con cui il sacro si mostra sullo schermo della coscienza, piuttosto che la sua spiegazione, rappresenta il fine dell'indagine. Da Otto prendono avvio gli studi fenomenologici sul sacro, da Durkheim quelli sociologici e culturali. Prima di esaminarli in rapporto allo studio degli spazi sacri, prendiamo in considerazione alcuni caratteri generali di questi ultimi, così come essi appaiono ad uno sguardo comparato sulla letteratura.

\subsection{Spazio sacro}

Sia esso santuario, chiesa, tempio, sacrario militare, nazionale o luogo legato ad altro valore culturale considerato fondamentale, lo spazio sacro è sempre separato dall'ambiente profano circostante. Si tratta di una separazione essenziale: «La vita religiosa e la vita profana non possono coesistere in uno stesso spazio. Affinché la prima possa svilupparsi, bisogna dunque assicurarle un luogo speciale, da cui l'altra venga esclusa. Da ciò ha origine l'istituzione di templi e santuari: essi rappresentano porzioni di spazio riservato alle cose e agli esseri sacri. [...] Queste sistemazioni sono [...] indispensabili a ogni vita religiosa» (Durkheim 2005/1912: 368).

Simboli materiali e prescrizioni rituali rimarcano la separatezza tra spazio sacro e profano (Eliade 2006/1957). Rito e luogo sacro sono indissociabili tra loro. Interdizioni e regole di purezza, culto, sacrificio, preghiera, meditazione, pellegrinaggio, cerimonie: lo spazio sacro è sempre uno spazio rituale.

La sacralità del luogo può essere spiegata da vari motivi. Uno sguardo alla storia comparata delle religioni evidenzia i seguenti: miti che ne fanno risalire l'origine all'azione divina; prossimità a luoghi in cui si è manifestato un segno soprannaturale; significativi eventi religiosi ivi avvenuti; associazione alla biografia di persone sante; presenza di reliquie o altri oggetti sacri; speciali configurazioni fisiche (cfr. Brereton 2005: 7.978ss.). Templi e santuari non esauriscono lo spazio sacro. Anche una casa può esserlo (Mazumdar, Mazumdar 1999). In una società mediatica, luoghi di pellegrinaggio non privi di sacralità sono legati a personaggi dello spettacolo: ne sono alcuni esempi Graceland, la 
villa di Elvis Presley, e la tomba di Jim Morrison al cimitero di Père-Lachaise (Margry 2008).

Da una prospettiva strettamente religiosa, sono generalmente riscontrabili tre funzioni dei luoghi sacri. Primo, permettere il contatto tra l'uomo e il divino; si trovano così simboli che rappresentano la divinità e ne sono canali di accesso. Secondo, accedere al potere sacro; ciò è evidente nei pellegrinaggi per richiedere l'intercessione divina. Terzo, esprimere l'ordine del mondo, contribuendo così a riprodurlo (Brereton 2005: 7.980ss.; Geertz 1957). In chiave non religiosa si possono leggere i luoghi sacri partendo da quest'ultima funzione, che in quanto connessa alla visione del mondo e ai valori di una cultura, è di per sé indipendente dalla sfera soprannaturale. Per esempio, in un sacrario nazionale non si è alla presenza di divinità, ma di valori sovra-individuali e inviolabili che rappresentano simbolicamente un ordine morale e una visione della vita civile della Nazione.

Si focalizza ora l'attenzione sui due approcci, prima introdotti, nella loro relazione con lo studio dello spazio sacro. L'approccio fenomenologico, dopo l'opera seminale di Otto, viene sviluppato da Gerardus van der Leeuw (1986/1933) e, in relazione allo spazio sacro, soprattutto da Mircea Eliade (2006/1957). In questo filone si trovano anche studi più recenti come quelli di Belden C. Lane (2001; 2002/1988). Il filone socio-costruzionista prosegue con numerosi studi, tra cui quelli di Jonathan Z. Smith (1972; 1992/1987), e di David Chidester e Edwar T. Linenthal (1995).

\subsection{L'approccio fenomenologico allo spazio sacro}

Per Eliade l'uomo ha bisogno del sacro perché prova il «desiderio di sistemarsi in una realtà oggettiva, di non venire paralizzato dal relativismo senza fine delle esperienze puramente soggettive» (2006/1957: 24). Il sacro si manifesta attraverso la realtà profana (ierofania), mostrando, tramite questa, una realtà totalmente diversa ed assoluta (Ganz Andere). Una ierofania può manifestarsi tanto mediante un uomo (ad es. Cristo), quanto mediante un oggetto inanimato (ad es. una pietra). Quando una ierofania si mostra, lo spazio originariamente omogeneo e privo di punti di riferimento si differenzia in spazio sacro e profano: «si richiede un segno per mettere fine alla tensione provocata dal relativismo e all'ansietà conseguente al disorientamento, insomma per trovare un punto d'appoggio assoluto» (ivi: 23 ). Il sacro non solo distingue, orizzontalmente, spazio sacro e profano, ma mette anche in comunicazione, verticalmente, mondo umano e soprannaturale. Il luogo della ierofania è "centro" verso cui orientarsi e axis mundi che collega Terra, Cielo e Inferi, spesso rappresentato da una montagna sacra o altro simbolo verticale.

Nelle società tradizionali, spiega Eliade, l'edificazione di villaggi, città, templi e case ripete un atto cosmogonico originario. In altri termini, ogni creazione spaziale ripete ritualmente la creazione del cosmo da parte degli dèi; di conseguenza, ogni spazio creato è imago mundi: «un Universo nasce dal suo 
proprio Centro, estendendosi dal punto centrale che è come il suo "ombelico". [...] A imitazione dell'Universo che si sviluppa dal Centro e si estende verso $\mathrm{i}$ quattro punti cardinali, il villaggio si costituisce» (ivi: $33 \mathrm{~s}$.). Se gli atti di creazione ripetono tutti il gesto della creazione originaria, i templi e santuari religiosi godono tuttavia di una particolare perfezione: non sono «soltanto una imago mundi, bensì anche la riproduzione sulla Terra di un modello trascendente [...] luogo santo per eccellenza, casa degli dèi, il Tempio santifica continuamente il Mondo [...] si trova al riparo da ogni corruzione terrestre, e ciò per il fatto che il progetto architettonico del Tempio è opera divina» (ivi: 42s.). Questo per quanto riguarda le società tradizionali, secondo Eliade. Oggi le cose sarebbero un po' diverse. Tuttavia, sebbene la società sia andata secolarizzandosi, l'uomo non diventa mai completamente profano. Anche al di fuori delle religioni istituite, «sussistono luoghi privilegiati, qualitativamente diversi dagli altri: il paese natale, il luogo dei primi amori [...] Tutti luoghi che, anche per l'uomo prettamente non-religioso, conservano una qualità eccezionale, "unica": sono "luoghi santi" del suo universo privato» (ivi: 21). Sono molti gli insegnamenti che si possono trarre da Eliade. Tra questi merita di essere sottolineato il ruolo di orientamento esistenziale che caratterizza il luogo sacro. In quanto centro simbolico di un "ordine del mondo", esso permette di reimmergersi nei valori che sono al cuore dell'identità personale e culturale. Si può quindi capire quanto significativa ed emozionante possa essere la visita ad un luogo sacro, e quanto esso possa essere considerato un bene prezioso da difendere.

Lane (2002/1988: 15), impegnato nello studio della spiritualità americana, riconosce grande importanza alle narrazioni: «without exception, the sacred place is the place rich in story». Particolarità di queste narrazioni è l'intreccio tra ierofania, cultura e soprattutto luogo, morfologicamente inteso e vissuto con i sensi e con la mente. La mitogenesi, in altri termini, non è totalmente arbitraria, ma viene evocata almeno in parte dalle caratteristiche fisiche (affordance) proprie dei luoghi stessi: «The place is "known" only to the extent that we participate in the various affordances it offers [...] An evocative landscape [...] seems to "call for" certain kinds of interpretation. Husserl used the word "intersubjectivity" in describing this process of reciprocal perception» (Lane 2001: 67). Come per Eliade, anche per Lane il sacro irrompe nella realtà, manifestandosi di sua iniziativa - «sacred place is not chosen, it chooses» (Lane 2002/1988: 19) ma la natura del luogo ha un ruolo nella sua espressione e così anche la cultura di chi lo percepisce: «The sacred site speaks, then, with its own voice, even as that voice is heard by thoroughly culturally conditioned ears. Furthermore, one only perceives the "other" (the numinous or holy) in connection with the site as this, too, is inescapably mediated by culture and place» (Lane 2001: 70).

\subsection{L'approccio socio-culturale allo spazio sacro}

L'approccio socio-culturale allo studio dello spazio sacro è stato anche definito, in opposizione a quello fenomenologico, nei termini di una politica a 
confronto con una poetica dello spazio sacro (Chidester, Linenthal 1995). Lane stesso (2001: 72ss.), effettivamente, ritiene che al ricercatore che studia i luoghi sacri possa essere utile la sensibilità del poeta. L'attenzione fenomenologica al vissuto esperienziale può certamente illuminare aspetti interessanti del rapporto tra uomo e luogo, tuttavia, rischia di lasciare sullo sfondo le complesse dinamiche sociali in cui lo spazio sacro può essere irretito e la cui individuazione sembra rappresentare, invece, il punto di forza dell'approccio sociologico e culturale. Questa differenza non va comunque esagerata: non mancano spunti politici nemmeno nella fenomenologia. Per esempio, gli stessi Chidester e Linenthal (1995: 5ss.) osservano che van der Leeuw (1986/1933) riconosce il potere intrinseco alla fondazione di un luogo sacro in quanto atto di conquista, di appropriazione, di negoziazione dell'accesso che prevede la scelta di criteri di inclusione e di esclusione. Analogamente, Eliade osserva che la conquista di un territorio si è sempre accompagnata alla sua consacrazione: «i conquistadores spagnoli e portoghesi si impadronivano, in nome di Cristo, dei territori che avevano scoperto e conquistato. L'erezione della Croce consacrava la regione, ed equivaleva in un certo senso a una "nuova nascita"» (2006/1957: 26). Infine, se si considera l'importanza del luogo sacro per "l'ordine del mondo", allora si comprende che «qualsiasi colpo infertogli dall'esterno rischia di trasformarlo in "Caos". E dal momento che "il nostro mondo" è stato fondato a imitazione dell'opera esemplare degli dèi [...], gli avversari sono paragonabili ai nemici degli dèi, i demoni» (ivi: 35 ). L'indagine sulla dimensione del potere non resta preclusa nemmeno all'approccio fenomenologico, dunque. Tuttavia, poiché l'approccio socio-culturale considera lo spazio sacro, fin da principio, come uno spazio socialmente prodotto, da questa prospettiva emergono in modo più diretto i suoi rapporti con il contesto sociale. Ciò non di meno, certi aspetti simbolici dei luoghi sacri, come quelli descritti da Eliade, possono essere integrati in questa visione. Per esempio, Roger Caillois utilizza la distinzione tra centro e periferia per illustrare l'organizzazione spaziale della società nello "spazio sacro" cittadino: «La configurazione delle città moderne rende ancora percepibile, a un certo livello, il valore in parte mitico, in parte oggettivo, di questa disposizione: al centro, la chiesa o la cattedrale (sede del divino) il municipio, gli edifici ufficiali [...] Intorno a questo nucleo rassicurante, caldo, ufficiale, i grandi agglomerati urbani sviluppano una cintura d'ombra e di miseria dove le strade sono strette, male illuminate, poco sicure, dove si trovano gli alberghi malfamati, i tuguri e i diversi tipi di edifici clandestini, dove si immagina si trovino riuniti vagabondi, prostitute, fuorilegge di ogni specie» (2001/1950: 46s.).

Tra i contributi più recenti in questo filone, si trovano, tra gli altri, quelli di Smith e dei già citati Chidester e Linenthal. Per Smith, allievo e critico di Eliade (Smith 1972), il rito crea il sacro e il luogo sacro è frutto di un lavoro di costruzione rituale, culturale e politica. Inoltre, il luogo sacro partecipa al processo di consacrazione rituale, funzionando come una focusing lens: «The temple serve as a focusing lens, establishing the possibility of significance by directing attention, by requiring the perception of difference. Within the temple, the or- 
dinary [...] becomes significant, become "sacred", simply by being there. A ritual object or action becomes sacred by having attention focused on it in a highly marked way. From such a point of view, there is nothing that is inherently sacred or profane. These are not substantive categories, but rather situational ones. Sacrality is, above all, a category of emplacement» (1992/1987: 104). La metafora delle lente mette anche in evidenza quale possa essere il potere simbolico che può derivare dal controllo di un luogo sacro. Questo è quanto viene messo al centro dell'attenzione da Chidester e Linenthal (1995), che nell'introduzione ad American Sacred Space illustrano una serie di categorie analitiche utili allo studio dello spazio sacro. Sono tre, secondo questi studiosi, le caratteristiche dei luoghi sacri: ritualizzazione, significazione e competizione in merito alla legittima proprietà del luogo. La ritualizzazione comprende culto, preghiera, pellegrinaggio e altri rituali che consacrano il luogo, nonché prescrizioni relative alle regole di purezza necessarie a restare degnamente nei suoi confini. Qualora queste non siano rispettate, si determina una profanazione causata da contaminazione, che può essere fronteggiata con riti di esclusione (allontanamento del reo) e purificazione rituale. Una seconda forma di profanazione è l'espropriazione. Questo è il caso, per esempio, di un antico sito sacro hawaiano, ancora sacro per i tradizionalisti locali ma "espropriato" dal governo americano che ne ha fatto un parco nazionale. La notte, quando i turisti se ne sono andati, i tradizionalisti entrano nel sito per riconsacrarlo: atto religioso, rituale, ma anche di riappropriazione. Scoppiata una contesa sulla legittima proprietà di questo luogo, esso è stato risignificato da sito religioso a potente simbolo del diritto all'autodeterminazione rivendicato dal popolo hawaiano.

Ricchezza e produttività simbolica sono una caratteristica degli spazi sacri: essi sono simboli identitari, rappresentano e permettono di riprodurre ritualmente valori e visioni del mondo di una società, ovverosia di ripassare $\mathrm{i}$ confini tra le categorie in cui vengono classificati eventi, persone e cose, assegnando loro una posizione nel sistema culturale e sociale. Di conseguenza, purezza e potere sono sempre in gioco quando ci si avvicina agli spazi sacri. Anche per questa ragione, lo spazio sacro può essere oggetto di contesa. Anzi, per Chidester e Linenthal è sempre conteso: «Sacred space is inevitably contested space, a site of negotiated contests over the legitimate ownership of sacred symbols. [...] Sacred places are arenas in which power relations can be reinforced, in which relations between insiders and outsiders, rulers and subjects, elders and juniors, males and females, and so on, can be adjudicated» (1995: $15 \mathrm{~s}$.).

Grado di contesa e sacralità percepita sembrano essere in relazione recipro$\mathrm{ca}$, così, tanto più il luogo sacro viene percepito a rischio di profanazione, tanto più la percezione della sua sacralità sembra aumentare. Chidester e Linenthal individuano quattro strategie utilizzate nella produzione dello spazio sacro: appropriazione, esclusione, inversione e ibridazione. Non si tratta di strategie solo simboliche ma anche politiche: «powerful, practical maneuvers in the field of sacred symbols» (ivi: 19). Le prime due sono strategie principalmente di pote- 
re, le ultime due di resistenza al potere. Nelle strategie di appropriazione, il potere si afferma rivendicando la legittima proprietà del luogo sacro, utilizzando anche strategie di esclusione per rafforzare questa rivendicazione in nome della purezza. Le strategie di inversione e ibridazione tendono invece all'innovazione, mescolando oppure rovesciando le relazioni simboliche dominanti, anche nelle loro espressioni spaziali (centro/periferia, alto/basso, dentro/fuori). Queste strategie di resistenza, secondo Chidester e Linenthal, sono comunque generalmente attive anche nelle costruzioni dominanti del sacro: «After all, Pearl Harbor itself is a hybrid of national park, patriotic monument, and military cemetery» (ibidem: 19$)$.

\section{Per concludere}

Caillois scriveva che «in fondo, del sacro in generale, la sola cosa che si possa affermare con sicurezza è compresa nella definizione stessa del termine: ciò che si contrappone al profano» (2001/1950: 9). Di certo, il concetto di sacro è complesso e comunica la sua complessità a quello di spazio sacro. Tuttavia, se è vero che il sacro si contrappone al profano così come il valore morale, non utilitaristico, assoluto e incontrattabile si oppone al valore utilitaristico delle cose quotidiane (Durkheim 2005/1912), allora si può anche affermare con sufficiente sicurezza che esso, proprio in quanto non negoziabile, può fornire un solido punto di riferimento esistenziale all'individuo e alla società (Eliade 2006/1957). Data la sua importanza simbolica e la sua connessione con l'identità personale e sociale, è anche comprensibile che il controllo dei luoghi sacri possa essere una fonte di capitale culturale e di potere che può essere oggetto di contesa, e che la profanazione del sacro e del luogo sacro rappresenti una grave minaccia, nonché una grave offesa morale, per l'identità personale $\mathrm{e}$ sociale di chi lo ha sacralizzato (Tetlock et al. 2000). Infatti, tanto il sacro rappresenta un punto fermo, tanto rischia di essere rigido e di scatenare facilmente il conflitto, qualora sia violato.

\section{Riferimenti bibliografici}

Belk R.W. et al. (1989), The Sacred and Profane in Consumer Behavior: Theodicy on the Odyssey, «The Journal of Consumer Research», 16(1), pp. 1-38.

Bellah R.N. (1967), Civil Religion in America, «Daedalus», 96(1), pp. 1-21.

Brereton J.P. (2005), "Sacred Space", in Jones L. (ed.), Encyclopedia of Religion, vol. 12 (second edition), Thomson Gale, Detroit.

Caillois R. (2001/1950), L'uomo e il sacro, Bollati Boringhieri, Torino.

Chidester D., Linenthal E.T. (1995), "Introduction", in Chidester D., Linenthal E.T. (eds), American Sacred Space, Indiana University Press, Bloomington and Indianapolis.

De Certeau M. (2001/1980), L'invenzione del quotidiano, Edizioni Lavoro, Roma. 
Durkheim É. (2005/1912), Le forme elementari della vita religiosa, Meltemi, Roma.

Eliade M. (2006/1957), Il sacro e il profano, Bollati Boringhieri, Torino.

Foucault M. (1986/1967), Of Other Spaces, «Diacritics», 16, pp. 22-27.

Gentile E. (2000), The Sacralisation of Politics: Definitions, Interpretations and Reflections on the Question of Secular Religion and Totalitarianism, «Totalitarian Movements and Political Religions», 1(1), pp. 18-55.

Kilde J.H. (2007), Space, Place, and Religious Meaning, «Material Religion», 3(2), pp. 277-278.

Knott K. (2010), Religion, Space, and Place. The Spatial Turn in Research on Religion, «Religion and Society: Advances in Research», 1, pp. 29-43.

Lane B.C. (2001), Giving Voice to Place: Three Models for Understanding American Sacred Space, «Religion and American Culture: A Journal of Interpretation», 11(1), pp. 53-81.

Lane B.C. (2002/1988), "Axioms for the Study of Sacred Place", in Lane B.C., Landscapes of the Sacred: Geography and Narrative in American Spirituality, The Johns Hopkins University Press, Baltimore - Maryland.

Lefebvre H. (1974), La production de l'espace, Antropos, Paris.

Luckmann T. (1969/1967), La religione invisibile, il Mulino, Bologna.

Margry P.J. (ed.) (2008), Shrines and Pilgrimage in the Modern World. New Itineraries into the Sacred, Amsterdam University Press, Amsterdam.

Mazumdar S., Mazumdar S. (1999), Women's Significant Spaces: Religion, Space, and Community, «Journal of Environmental Psychology», 19(2), pp. 159-170.

Otto R. (2009/1936), Il sacro, SE, Milano.

Pignato C. (2001), Totem mana tabù. Archeologia di concetti antropologici, Meltemi, Roma.

Prebish C.S. (1984), Heavenly Father, Divine Goalie: Sport and Religion, «The Antioch Review», 42(3), pp. 306-318.

Smith J.Z. (1972), The Wobbling Pivot, «The Journal of Religion», 52(2), pp. 134149.

Smith J.Z. (1992/1987), To Take Place: Toward Theory in Ritual (Chicago studies in the history of Judaism), The University of Chicago Press, Chicago and London.

Tetlock P.E. et al. (2000), The Psychology of the Unthinkable: Taboo Trade-offs, Forbidden Base Rates and Heretical Counterfactuals, «Journal of Personality and Social Psychology», 78(5), pp. 853-870.

Timothy D.J., Olsen D.H. (2006), "Tourism and Religious Journeys", in Timothy D.J., Olsen D.H. (eds), Tourism, Religion and Spiritual Journeys, Routledge, New York.

Trombetta P.L. (2004), Il bricolage religioso. Sincretismo e nuova religiosità, Dedalo, Bari.

van der Leeuw G. (1986/1933), Religion in Essence and Manifestation, Princeton University Press, Princeton. 